\title{
Dissociating crossmodal and verbal demands in paired associate learning (PAL): What drives the PAL-reading relationship?
}

\author{
Robin A. Litt ${ }^{\mathrm{a}^{\mathrm{*}}}$, Peter F. de Jong ${ }^{\mathrm{b}}$, Elsje van Bergen ${ }^{\mathrm{ab}}$, and Kate Nation ${ }^{\mathrm{a}}$ \\ ${ }^{a}$ Department of Experimental Psychology, University of Oxford, South Parks Road, Oxford \\ OX1 3UD, United Kingdom \\ ${ }^{\mathrm{b}}$ Research Institute of Child Development and Education, University of Amsterdam, The \\ Netherlands
}

*Corresponding author.

Email address: $\underline{\text { robin.litt@psy.ox.ac.uk }}$

Telephone: +447411606063

Fax: +44 (0) 1865310447

Please cite this article as:

Litt, R.A., de Jong, P.F., van Bergen, E., \& Nation, K. (2013). Dissociating crossmodal and verbal demands in paired associate learning (PAL): What drives the PAL-reading relationship? Journal of Experimental Child Psychology, 115(1), 137-149.

http://dx.doi.org/10.1016/j.jecp.2012.11.012 


\begin{abstract}
Recent research suggests that visual-verbal paired associate learning (PAL) may tap a crossmodal associative learning mechanism that plays a distinct role in reading development. However, evidence from children with dyslexia indicates that deficits in visual-verbal PAL are strongly linked to the verbal demands of the task. The primary aim of this study was to disassociate the role of modality and verbal demand in driving the PAL-reading relationship. To do so, we compared performance across four PAL mapping conditions: visual-verbal, verbal-verbal, visual-visual, and verbal-visual. We reasoned that if crossmodal mapping demand accounts for the PALreading relationship, both visual-verbal and verbal-visual PAL should exhibit significant relationships with reading ability. The results were incompatible with the crossmodal hypothesis. Only tasks requiring verbal output (visual-verbal, verbalverbal PAL) significantly correlated with reading ability. Additionally, visual-verbal and verbal-verbal PAL were well represented by a latent "verbal-output PAL" factor. Structural equation modeling showed that this factor fully accounted for the PALreading relationship; visual-verbal PAL did not add anything to the prediction of reading above and beyond this latent factor. The results are interpreted according to an alternative verbal account of the PAL-reading relationship.
\end{abstract}

Keywords:

Paired associate learning

Crossmodal associations

Rapid automatized naming

Phonological awareness 
Dissociating crossmodal and verbal demands in paired associate learning (PAL):

What drives the PAL-reading relationship?

Two of the skills most strongly associated with reading development are phonological awareness (e.g., Melby-Lervag, Lyster \& Hulme, 2012) and rapid automatized naming (RAN; e.g., Norton \& Wolf, 2012). In recent years, paired associate learning (PAL) has garnered increasing interest as an additional, independent predictor of reading ability (Hulme, Goetz, Gooch, Adams, \& Snowling, 2007; Warmington \& Hulme, 2012; Windfur \& Snowling, 2001).

PAL involves learning arbitrary associations between stimulus (i.e., input) items and response (i.e., output) items in memory. These pairings can be unimodal (e.g., visual-visual, verbal-verbal) or crossmodal (e.g., visual-verbal) in nature. In the context of reading development, crossmodal PAL is here defined as any association requiring a connection (regardless of the direction) between a visual and a verbal stimulus. Crucially, performance on any PAL task depends on successful learning of three distinct components: the stimulus item, the response item, and the association between the two. Thus, individual differences in performance may stem from processes operating at any of these three levels. Reading, especially in the early stages of development, may be classed as a form of crossmodal, visual-verbal PAL. Learning the letters of the alphabet, for example, depends on the formation of arbitrary sound-symbol associations. Letter knowledge is widely viewed as a precursor to the alphabetic principle and the extent of the child's letter knowledge is a robust predictor of reading ability (Byrne, 1998; Hulme et al., 2007; Lervåg, Bråten, \& Hulme, 2009; de Jong \& van der Leij, 1999; Muter, Hulme, Snowling, \& Stevenson, 2004; Schatschneider, Fletcher, Francis, Carlson, \& Foorman, 2004). Additionally, in deep orthographies such as English, the acquisition of irregular sight 
words may rely in part on this ability to form arbitrary orthography-to-phonology mappings (Hulme et al., 2007; Windfuhr \& Snowling, 2001).

The potential involvement of visual-verbal PAL in reading fits well within a connectionist framework. In connectionist models, initially arbitrary connections between orthographic input and phonological output are strengthened through a learning algorithm that alters connection weights through repeated experience. These connection weights are viewed as analogous to the associations formed between visual and verbal stimuli in a PAL task (Hulme et al., 2007; Seidenberg \& McClelland, 1989; Warmington \& Hulme, 2012; Windfuhr \& Snowling, 2001). Importantly, varying the efficiency of this learning mechanism (by altering the rate with which connection weights are altered), or limiting its resources (by removing hidden units), results in a differential pattern of impairment than degrading the phonological representations, suggesting unique roles for these mechanisms in reading development (Harm \& Seidenberg, 1999; Seidenberg \&McClelland, 1989).

Consistent with this pattern of findings, studies in typically developing readers have repeatedly shown that visual-verbal PAL is independent from phonological awareness and a strong concurrent predictor of reading ability (Hulme et al., 2007; Warmington \& Hulme, 2012; Windfuhr \& Snowling, 2001). Similarly, although PAL and RAN correlate moderately with each other, they tap separate mechanisms involved in reading (Lervåg, Bråten, \& Hulme, 2009). Warmington and Hulme (2012) reported that even after accounting for phoneme deletion and RAN, visual-verbal PAL was a significant predictor of word and nonword reading accuracy, as well as reading speed. Together, these findings suggest that visual-verbal PAL is an independent predictor of reading development, accounting for variance in both lexical and nonlexical components of reading ability. 
However, the underlying mechanisms responsible for the involvement of visual-verbal PAL in reading have yet to be fully elucidated. A recent theoretical account of the PAL-reading relationship comes from Hulme and colleagues, who proposed that visual-verbal PAL taps a crossmodal associative learning mechanism involved in establishing orthography-phonology mappings (Hulme et al., 2007; Warmington \& Hulme, 2012). This crossmodal hypothesis comes from a study in which the authors compared three PAL tasks (visual-verbal, visual-visual, and verbalverbal) to evaluate the specificity of the relationship between visual-verbal PAL and reading (Hulme et al., 2007). The inclusion of verbal-verbal PAL in the design marked a necessary step in understanding the PAL-reading relationship. In requiring verbal learning and verbal output, both visual-verbal PAL and verbal-verbal PAL tax the verbal system, whereas visual-visual PAL provides a proxy of more general associative learning abilities. Given that verbal skills, such as verbal short term memory and phonological awareness, are strongly implicated in reading development, one would expect tasks that draw on this system to be related to reading ability (e.g., Snowling \& Hulme, 1994; Vellutino, Fletcher, Snowling, \& Scanlon, 2004).

Consistent with this idea, both visual-verbal and verbal-verbal PAL correlated with reading ability, whereas visual-visual PAL did not (Hulme et al., 2007). Although Hulme and colleagues noted that visual-verbal PAL appeared to have a slight edge over verbal-verbal PAL in the strength of its correlations with reading, the significance of this difference was never tested. However, visual-verbal PAL was the only PAL task to predict unique variance in reading ability above and beyond phoneme deletion and the other PAL measures. These findings were interpreted as evidence for the specific role of visual-verbal PAL in reading, leading the authors to 
suggest that visual-verbal PAL taps modality specific "connection forming" mechanisms, akin to those operating in connectionist models.

Despite the appeal of the crossmodal hypothesis for theories of reading development, there is an alternative account of PAL that warrants further investigation. We term this the verbal account. The basis for this account stems from findings in children with dyslexia, in which deficits in visual-verbal PAL appear to be related to the verbal nature of the task, rather than its crossmodal demands (Mayringer \& Wimmer, 2000; Messbauer \& de Jong, 2003; Vellutino, Steger, Harding, \& Phillips, 1975; Vellutino, Scanlon, \& Spearing, 1995; Wimmer et al., 1998). For example, Mayringer and Wimmer (2000) showed that children with dyslexia are impaired in visual-verbal PAL when the verbal stimuli comprise nonwords, but not when they comprise short, familiar words. In Velluntio et al.'s (1975) study, children learned two crossmodal associations: visual-verbal and visual-auditory (termed "nonverbal"). The first required an association between a visual symbol and a nonword, whereas the latter required an association between a visual symbol and a nonverbal oral sound (e.g., humming, coughing, puckering lips). Although both experimental conditions required a crossmodal association, as well as oral production, children with dyslexia exhibited impairment only in visual-verbal PAL. Thus, the dyslexia literature is strongly suggestive of a primary difficulty with the verbal component of visual-verbal PAL rather than the formation of crossmodal associations. Indeed, many researchers have proposed that verbal learning is the crucial component of visual-verbal PAL, because the most robust deficits are observed for nonword stimuli (Elbro \& Jensen, 2005; Mayringer \& Wimmer, 2000; Vellutino et al., 1975). But what role might verbal learning play in reading development? 
Although children come to the task of reading with established phonological representations (formed through oral language experience), the acquisition of novel phonological forms (i.e. new vocabulary) continues via written language experience once the child is literate. In addition to this more obvious case of verbal learning, recent evidence suggests that the process of learning to read requires the acquisition of a less intuitive class of novel phonological representations (Elbro \& Jensen, 2005; Elbro, de Jong, Houter, \& Nielsen, 2012; Ranbom \& Connine, 2011; Tunmer \& Chapman, 2011). These representations can be seen as orthographically derived (resulting from written rather than oral language experience) (Ranbom \& Connine, 2011). Consider the following illustration: when a child encounters an irregular word such as yacht for the first time, he or she will attempt to decode it using graphemephoneme correspondence rules. The first attempt (and likely several subsequent attempts) will inevitably lead to an incorrect pronunciation "y-a-ch-t." Here, the pronunciation derived from the decoding attempt clearly differs from the spoken pronunciation. So how does a child learn that "yacht" is in fact "yot"?

One suggestion is that the spelling pronunciation (the pronunciation generated via phonological recoding) becomes linked to the phonological representation of the spoken word (Elbro et al., 2012; Ranbom \& Connine, 2011; Tunmer \& Chapman, 2011). This process, akin to verbal-verbal PAL, allows the novice reader to utilize the product of phonological recoding to arrive at the correct spoken word representation. Evidence for this comes from Elbro et al., (2012), who found that children's ability to recognize words from spelling pronunciations accounted for unique variance in reading ability, even after controlling for phoneme deletion, RAN, and vocabulary. This skill was equally important for regular and irregular word reading, which was explained by the fact that for beginning readers, the product of a decoding attempt 
may be quite far from the spoken form, even for regular words (e.g., "cuuuh...aaa..tuuh" is not equivalent to "cat"). Thus the acquisition of novel phonological representations may be a general requirement of learning to read. It is this verbal learning ability that may in fact be indexed by visual-verbal PAL.

Clearly, there is an alternative theoretical framework from which to interpret the PAL-reading relationship. The aim of the current study was to directly evaluate the crossmodal and verbal accounts of the PAL-reading relationship by dissociating the role of modality and verbal demands. To do so, we included four PAL conditions: visual-verbal, verbal-verbal, visual-visual, and verbal-visual. The novel crossmodal condition, verbal-visual PAL, was included for two reasons. First, it allowed for a fully balanced design in which each of the four mapping conditions is uniquely characterized by both modality (crossmodal, unimodal) and output demand (visual, verbal). This provided the comparisons necessary to directly test both the crossmodal account and the alternative verbal account of the PAL-reading relationship. Second, although Hulme and colleagues' (2007) theory does not specifically address mapping direction, its focus on the level of the association (rather than stimulus-specific or response-specific learning) naturally leads to the prediction that any task requiring an association between a visual and verbal stimulus should be involved in reading development. The addition of verbal-visual PAL to our design therefore addressed the prediction that both crossmodal tasks should correlate with reading, regardless of the direction of the mapping.

We hypothesized that if crossmodal associative learning is the primary mechanism underlying the PAL-reading relationship, both visual-verbal PAL and verbal-visual PAL should demonstrate strong predictive relationships with reading, independent of the effects of phoneme deletion and RAN. Crucially, although one 
might expect differences in the strength of the relationship as a function of task similarity (e.g., mapping direction is the same for visual-verbal PAL and reading, but different for verbal-visual PAL and reading), mapping direction should not fundamentally determine the PAL-reading relationship. Instead, the shared "crossmodal" component of visual-verbal and verbal-visual PAL (as tapped by a latent variable) should fully account for the PAL-reading relationship. On the other hand, if verbal demand drives the PAL-reading relationship, both visual-verbal PAL and verbal-verbal PAL should show strong predictive relationships with reading, independent of the effects of phoneme deletion and RAN. Additionally, the shared "verbal output" component of visual-verbal and verbal-verbal PAL (as tapped by a latent variable) should fully account for the PAL-reading relationship.

\section{Method}

\section{Participants}

Sixty-four children (30 male, 34 female) ranging in age from 7 years, 9 months to 11 years, 9 months $(\mathrm{M}=9$ years, 7 months; $\mathrm{SD}=9.30$ months $)$ were recruited from three state primary schools serving socially-mixed catchment areas in Oxfordshire, UK. All children scored within average range (T score between 40 and 60) on the Matrix Reasoning subtest from the Wechsler Abbreviated Scale of Intelligence (WASI; Wechsler, 1999).

\section{Design}

Children were tested in five sessions across a five-week period. In week one, children completed tests of nonverbal reasoning, reading ability, phonological awareness, and rapid automatized naming. In sessions 2-5 children completed each of the four PAL mapping conditions. One condition was tested per week to minimize 
interference between conditions and the order in which children completed the conditions was counterbalanced.

\section{Materials and Procedure}

Reading and reading-related skills. The following standardized assessments were made with the tests administered according to the test manual instructions.

Single Word Reading. The Single Word Reading Test (SWRT) 6-16 was administered as a measure of untimed single word reading ability (Foster, 2007). Children are instructed to read aloud as many words as possible, starting at the top of the page. There is no time limit on this test.

Word Reading Fluency. The Sight Word Efficiency subtest from the Test of Word Reading Efficiency (TOWRE; Torgesen, Wagner, \& Rashotte, 1999) was used as a measure of sight word reading. The raw score is defined as the total number of words (out of 104) that can be read correctly in 45 seconds.

Nonword Reading Fluency. The Phonemic Decoding Efficiency subtest from the TOWRE (Torgesen et al., 1999) was used as a measure of speeded nonword reading. The raw score is the total number of nonwords read correctly in 45 seconds.

Phoneme Deletion. The Elision subtest of the Comprehensive Test of Phonological Processing (CTOPP; Wagner, Torgesen, \& Rashotte, 1999) assessed phonological awareness (PA). Participants are asked to delete single phonemes from words (e.g., "Say bold without the b").

Rapid Automatized Naming. The rapid digit naming subtest of the CTOPP was administered as an assessment of RAN. Six digits (e.g., 4, 7, 8, 5, 2, 3) are repeated six times throughout a 36-item matrix arranged in four rows of nine items. Children must name the items in the matrix sequentially, starting at the top of the page from 
left to right as quickly as possible until they have named all 36 items. Children complete two separate arrays that differ only in the order the digits are presented. The time (in seconds) taken to name the items in each array is summed to produce a total naming score.

Paired-associate learning. All children completed four PAL mapping conditions: visual-verbal, verbal-verbal, visual-visual, and verbal-visual. For each condition they learned six stimulus-response pairs comprised of sets of verbal and/or visual stimuli. The stimulus-response pairs were fixed, such that all children learned the same pairings. The presentation of the stimulus-response pairs was randomized for each trial.

Visual Stimuli. The abstract symbols within each PAL condition were chosen from extinct written languages (e.g., Akkadian) and matched across conditions for difficulty of verbalization. These data were obtained from testing in which 15 adults were asked to rate 54 potential stimulus items on a scale of 1-10 (with 10 being most difficult) for degree of verbalization (i.e., "how easy is it to assign a verbal label or description to this item?"), and ease of drawing (i.e., "how easy is it to draw this item?"). The 24 symbols hardest to verbalize were assigned to four sets (i.e., two sets of stimulus items, and two sets of response items) that comprised the visual stimuli for the PAL conditions. The visual stimuli were matched for ease of verbalization across the four sets, $F(3,42)=2.11, p=.114$. Additionally, we ensured that the two sets comprising visual response items were matched for ease of drawing, $F(1,14)=$ $1.26, p=.280$.

Verbal Stimuli. The verbal stimuli comprised nonwords, all phonotatically legal CVC strings, chosen from the ARC Nonword Database (Rastle, Harrington, \& Coltheart, 2002). Each of the short vowel sounds (i.e., a, e, i, o, u) and one long vowel 
sound (e.g., ee) were used once per stimuli set. Additionally, no two items shared consonant positioning (i.e., first or last sound) within a stimuli set in an effort to minimize phonological similarity between items.

PAL Procedure. Children were tested individually in a quiet room in their school. The experiment was run using EPrime Version 2.0 presented on a Dell laptop. In each condition, the six stimulus-response pairs were presented in two presentation trials before the test trials began. In the first presentation trial, children were instructed to repeat the response item after each presentation to ensure that the responses could be accurately reproduced. For verbal response items, children were instructed to repeat each nonword aloud; for visual response items, children were instructed to draw the abstract symbol inside a booklet of blank index cards, one item per page to prevent them from referring to previous responses throughout a trial. Rarely ( $<5 \%$ of first presentation to a response item), a nonword or symbol was reproduced incorrectly. In this case, the experimenter corrected the child by saying "that's not quite right," and then proceeded to repeat the correct nonword or draw the correct response in the booklet. In the second presentation trial the children were asked to focus on learning the pairs of items and not to reproduce the response item (following pilot testing in which instructing participants to focus on the stimulusresponse pairings resulted in better learning than instructing them to reproduce the response items).

Five blocks of learning trials followed the presentation trials. Each block consisted of six trials, one for each stimulus-response pair. In each trial, the stimulus item was presented on the computer and the participant was then asked to provide the appropriate response (e.g., "What goes with hib?"). Depending on the output required, the child either drew the symbol in the booklet or verbally produced the nonword that 
corresponded to the stimulus item. Regardless of the accuracy of the response, the correct answer was presented on the computer immediately following each trial. This procedure was repeated for each trial across all five blocks. All participants completed all trials, and accuracy per trial and total learning across trials was recorded. The final score was calculated as the total number of correct trials across all five blocks (maximum score of 30).

\section{Results}

The children performed at an average level for reading, phonological awareness and RAN, according to population norms (see Table 1). The scores of two children (one for visual-verbal PAL and one for verbal-verbal PAL) were excluded pairwise from all analyses, as the scores were $>4 S D s$ above the sample mean. Excluding these values did not alter the pattern of results. Although visual-verbal PAL and verbalverbal PAL demonstrated slight positive skewness, the score distributions for all measures were normally distributed.

As indicated by the relatively modest mean scores across PAL conditions, the children found the PAL tasks quite challenging. Despite this, it is clear that our tasks captured a wide range of performance across all four PAL conditions. A repeatedmeasures analysis of variance showed that performance varied significantly across PAL conditions, $F=40.70, p<.001, \eta_{p}{ }^{2}=.39$. Planned pairwise comparisons (with Bonferroni correction) revealed two interesting findings. First, there were no significant differences between conditions with the same output demand. That is, there were no significant differences between the two verbal output conditions, visualverbal PAL and verbal-verbal PAL, $p=.639$, and no significant differences between the visual output conditions, verbal-visual and visual-visual PAL, $p=.198$. Second, comparisons within modality revealed a significant difference between the 
crossmodal conditions (visual-verbal, verbal-visual), $p<.001$, as well as a significant difference between the unimodal conditions (verbal-verbal, visual-visual), $p<.001$. In both of these comparisons, performance was lowest in the condition requiring verbal output. Thus, performance differences appear to be driven by the increased difficulty of the two verbal output conditions.

\section{Relationship between PAL and reading measures}

Table 2 displays the simple correlations (above the diagonal) and partial correlations controlling for age (below the diagonal) among measures. The agecontrolled correlations are slightly lower than the simple correlations, but show the same overall patterns. As there was no difference in the pattern of correlations, we focus here on the age-controlled correlations

As expected, the reading measures correlated highly with each other.

Performance across the four PAL conditions was moderately correlated $(r=.29-.42)$. The pattern of the correlations between the PAL tasks and reading is striking: only visual-verbal PAL and verbal-verbal PAL demonstrate significant correlations with reading. In contrast, visual-visual PAL and verbal-visual PAL demonstrate a clear lack of relationship with reading. The pattern of correlations indicates that verbal output, rather than crossmodal mapping, is a necessary component of the PALreading relationship — the verbal output PAL measures (visual-verbal, verbal-verbal) show similar and significant correlations with all three measures of reading (word fluency, nonword fluency, word accuracy), whereas the visual output PAL measures (visual-visual, verbal-visual) show nonsignificant correlations with all three measures. Modeling the relationship between PAL, phoneme deletion, RAN, and reading

Our next set of analyses addressed whether performance on the PAL tasks was associated with unique variance in reading, beyond the variance accounted for by 
phoneme awareness and RAN. We utilized maximum likelihood estimation techniques to conduct a series of path analyses using MPlus (version 5.21, Muthén \& Muthén, 2009). All raw scores on measures were residualized for age before running the analyses to enable the examination of age-independent relationships. To establish the strength of each task on its own as a predictor of reading, four separate models were constructed, one for each PAL task (Figure 1); including all tasks in the same model might have led to potential path inflation due to suppression effects, given the correlation between the PAL measures. The models allowed for correlations between predictors and all possible paths from the predictors (PAL, phoneme deletion and RAN) to reading (word accuracy, word fluency and nonword fluency).

Unsurprisingly, the paths from phoneme deletion and RAN to all three reading measures were significant in all models. In accordance with the verbal account of the PAL-reading relationship, both visual-verbal PAL (panel A) and verbal-verbal PAL (panel B) predicted significant unique variance in word reading accuracy. In contrast, visual-visual PAL (panel C) and verbal-visual PAL (panel D) did not contribute to the prediction of reading ability above and beyond phoneme deletion and RAN. Given the demonstrated lack of relationship with reading ability, these visual-output PAL tasks will not be considered in further analyses.

The similarity of the verbal-output PAL models is striking; both PAL measures predicted significant unique variance in word reading accuracy only, whereas phoneme deletion and RAN predicted unique variance in all three reading measures. However, although the structure of the models is identical, it is worth noting that the path weights from visual-verbal PAL to word and nonword fluency are marginal, and larger than those from verbal-verbal PAL to fluency measures. This may be suggestive of a difference in the strength of the verbal-output PAL tasks as 
predictors. If visual-verbal PAL makes a contribution to the prediction of reading fluency that cannot be accounted for by the verbal output component of the task, it would be inconsistent with a purely verbal account of the PAL-reading relationship.

\section{Examining the specificity of the PAL-reading relationship}

Having demonstrated that the PAL-reading relationship holds only for verbal output PAL tasks, we next evaluated whether this relationship can be fully accounted for by the shared component of the tasks. Additionally, we examined whether this shared "verbal-output PAL" component accounted for unique variance in reading, after controlling for phoneme awareness and RAN. We started by running a confirmatory factor analysis (CFA) with three factors: verbal-output PAL, phoneme deletion, and RAN. Our primary aim was to examine whether visual-verbal PAL and verbal-verbal PAL can be represented by an underlying latent factor, "verbal-output PAL." For the proper comparison of possible differences in the relationships between PAL, phoneme deletion, and RAN, we also specified latent variables for phoneme deletion and RAN. Doing so allowed us to account for differences in reliability between the tasks. To form latent factors for phoneme deletion and RAN, the overall scores were split into two sets of scores to be used as indicators. For phoneme deletion, scores were split into total number correct on odd and even test items. For RAN, we used the two subtests that comprised the total RAN score as indicators. This method resulted in two indicators for each latent variable. We ran a confirmatory factor analysis (CFA) with three factors: verbal-output PAL, phoneme deletion, and RAN. To aid in local identification, the indicators for each latent variable were specified with equal factor loadings. The results of the CFA indicated that a threefactor model fitted the data well, $\chi^{2}(9)=7.18, p=.619, \mathrm{RMSEA}=.000[90 \% \mathrm{CI}$ : $.000-.119], \mathrm{SRMR}=.04, \mathrm{CFI}=1.00$. The factor loadings for verbal-output PAL (.67), 
phoneme deletion (.93), and RAN (.94), were all significant at the $\alpha=.01$ level (Stevens, 2009).

Having confirmed that visual-verbal PAL and verbal-verbal PAL are well represented by an underlying latent variable, we next constructed a structural equation model ${ }^{1}$ with the three latent variables as predictors of word fluency, nonword fluency, and word accuracy. The model (Figure 2) provided an excellent fit to the data, $\chi^{2}(18)$ $=18.64, p=.415, \mathrm{RMSEA}=.024[90 \% \mathrm{CI}: .000-.115], \mathrm{SRMR}=.03, \mathrm{CFI}=.998$. Verbal-output PAL accounted for unique variance only in reading accuracy, whereas phoneme deletion and RAN accounted for unique variance in all three reading measures. Together, the latent variables accounted for $50 \%$ of the variance in word reading fluency, $58 \%$ of the variance in nonword reading fluency, and $53 \%$ of the variance in word reading accuracy.

To test whether visual-verbal PAL shares an additional and specific relationship with reading that cannot be accounted for by the latent construct (i.e., the shared component of visual-verbal PAL and verbal-verbal PAL) we ran the model with an added direct path from visual-verbal PAL to each of the reading measures (in separate iterations). The added direct effects did not result in significant improvement to model fit (word fluency, $\Delta \chi^{2}(1)=.71, p=.399$; nonword fluency, $\Delta \chi^{2}(1)=1.03, p=$ .310 ; word accuracy, $\left.\Delta \chi^{2}(1)=.61, p=.435\right)$. Thus, visual-verbal PAL did not account for variance in reading ability above that explained by the shared variance with verbal-verbal PAL.

\footnotetext{
${ }^{1}$ Structural equation modeling (SEM) is often used to determine the fit of a full causal model to the data, thus requiring a large sample size to ensure adequate power. However, in the current study SEM was used to perform multivariate multiple regression using latent independent variables. This is akin to multiple regression analyses in which one coefficient is tested to deviate significant from zero. In such analyses, a sample size of 64 is generally considered adequate to reject the null-hypothesis.
} 
Finally, we ran a series of constraints on the model to examine the differential importance of each latent variable to the prediction of fluency compared with accuracy. Constraining RAN to make equal contributions to word and nonword fluency did not affect model fit, $\Delta \chi^{2}(1)=.00, p=1.00$, indicating that it is an equally strong predictor of both fluency measures. In contrast, additionally constraining the fluency measures to be equal to accuracy resulted in significantly poorer model fit, $\Delta \chi^{2}(1)=10.16, p=.001$, indicating that RAN makes a stronger contribution to fluency than accuracy. For phoneme deletion, model fit was not significantly affected by imposing constraints on word and nonword fluency, $\Delta \chi^{2}(1)=1.34, p=.247$, or by the subsequent constraint between fluency and accuracy, $\Delta \chi^{2}(1)=1.91, p=.16$. Finally, for verbal-output PAL, model fit was not significantly affected by constraining word and nonword fluency to be equal, $\Delta \chi^{2}(1)=.04, p=.842$, or by the additional constraint between fluency and accuracy, $\Delta \chi^{2}(1)=1.26, p=.262$.

\section{Discussion}

The primary aim of this experiment was to evaluate the crossmodal account of the PAL-reading relationship. We examined performance across four PAL mapping conditions designed to dissociate the role of modality and verbal demands in determining the PAL-reading relationship. In line with Hulme et al. (2007) we found that both visual-verbal and verbal-verbal PAL correlated significantly with reading and phoneme deletion. Not surprisingly, visual-visual PAL was unrelated to reading ability; neither though was there a relationship between verbal-visual PAL and reading. This latter finding in regard to verbal-visual PAL is incompatible with the crossmodal hypothesis. Both visual-verbal and verbal-visual PAL required the crossmodal associations implied by Hulme and colleagues (2007) to drive the PALreading relationship, yet verbal-visual PAL was unrelated to reading ability. Instead, 
the results favored the alternative verbal account that the PAL-reading relationship is driven by mechanisms specifically involved in learning, accessing, and producing verbal material.

Before accepting this conclusion we need to ask whether the failure to detect a relationship between verbal-visual PAL and reading resulted from differences between the crossmodal tasks. Although there were differences in performance levels between conditions, we find it difficult to argue that verbal-visual PAL did not adequately tap crossmodal mapping mechanisms, or that the two crossmodal PAL tasks tapped these mechanisms in different ways. First, whereas low performance levels on visual-verbal PAL may signal concerns about task difficulty, performance levels on verbal-visual PAL (see Table 1) were ideal. Additionally, the range of scores on the tasks were essentially the same (visual-verbal: 0-25; verbal-visual: 027), so it cannot be argued that either task failed to capture the full range of performance necessary to detect a relationship with reading. Finally, performance was lower in both verbal output PAL conditions compared to visual output PAL conditions. Thus, the difference in performance levels between the crossmodal tasks was likely due to the targeted differences in verbal demand, rather than unintentional differences in crossmodal demand. One might even say that verbal-visual PAL, with its lesser demands on verbal output, provided a purer index of crossmodal associative learning. It is therefore unlikely that this task would fail to correlate with reading skill if in fact crossmodal mapping ability drives the PAL-reading relationship.

Having demonstrated that the verbal-output PAL tasks show a similar pattern of correlations with reading, we next addressed the two remaining criteria for acceptance of our verbal hypothesis. First, consistent with previous research, we found that visual-verbal PAL predicted significant variance in reading accuracy, 
above and beyond phoneme deletion and RAN (Hulme et al., 2007; Warmington \& Hulme, 2012; Windfuhr \& Snowling, 2001). Crucially, however, this finding also held for verbal-verbal PAL. Both tasks predicted significant variance in accuracy, but failed to reach significance for fluency. Although visual-verbal PAL showed a slightly stronger relationship with reading fluency than verbal-verbal PAL, this difference in relationship was not significant.

Second, in the latent model summarized in Figure 2, visual-verbal and verbalverbal PAL well represented by an underlying latent construct and, importantly, this construct fully accounted for the relationship between both PAL tasks and reading ability. That is, there was no evidence that visual-verbal PAL predicted extra variance in any of the reading measures once the latent construct was accounted for in the model.

This finding differs from that of Hulme and colleagues (2007). Whereas the pattern and strength of the specific correlations with the reading measures were largely equivocal in the current study, visual-verbal PAL exhibited slightly stronger (but not entirely systematic) correlations than verbal-verbal PAL in Hulme et al.'s (2007) study. Although the reasons for these differences are not entirely clear (perhaps due to differences in task reliability or adminstration procedures), it is unlikely to be due to the crossmodal demands of the task: verbal-visual PAL was unrelated to reading ability in the current study. Additionally, verbal-output PAL predicted unique variance in reading ability after controlling for the robust contribution of phoneme deletion and RAN, a finding that is consistent with the literature on visual-verbal PAL (Hulme et al., 2007; Warmington \& Hulme, 2011; Windfuhr \& Snowling, 2001). The fact that this finding held for our latent construct strongly suggests that verbal demand, rather than crossmodal demand, is responsible 
for the PAL-reading relationship. The latent construct necessarily accounted only for the verbal demands shared by visual-verbal and verbal-verbal PAL, thus we would not expect the results of our study to replicate those cited above if crossmodal demand were essential to the PAL-reading relationship. In all, we believe that we have strong evidence to assert that verbal demands drive the PAL-reading relationship.

How, then, should we conceive of the verbal demands of the tasks? What does verbal-output PAL measure and why is it related to reading ability? In line with previous research, the children in our sample did not exhibit varied performance in the immediate repetition of the nonwords (Mayringer \& Wimmer, 2000). This strongly indicates that individual differences in learning the paired associates arose not from immediate perceptual or working memory processes, but from later stages of processing involved in encoding, storage, retrieval, and /or output.

One possible explanation for our findings is that both reading and verbaloutput PAL depend upon successful access and retrieval of a phonological form. Consistent with this, studies have implicated deficient output processes in dyslexia (e.g., Elbro, 1998; Nation, Marshall, \& Snowling, 2001; Ramus \& Szenkovits, 2008; Snowling \& Hulme, 1994; Swan \& Goswami, 1997; Truman \& Hennessey, 2006). Such difficulties are typically only apparent for tasks in which the verbal system is sufficiently taxed and explicit access and output required (see Ramus \& Szenkovits, 2008). For example, children with dyslexia exhibit picture naming deficits when the stimuli comprise long, phonologically complex words, or words that are low in frequency, but not when they comprise short, high-frequency words (Swan \& Goswami, 1997; Nation et al., 2001). Although verbal-output PAL tasks clearly place strong explicit output demands on the verbal system, the fact that our stimuli comprised nonwords proves difficult for this explanation. Arguably, the processes 
involved in the access and retrieval of words from long-term memory differ from those utilized for recently learned nonwords (e.g., Davis, Di Betta, Macdonald, \& Gaskell, 2008; Dumay \& Gaskell, 2007).

Instead, it is likely that the verbal demands indexed by our PAL measures hinge critically on a skill inherent to nonword tasks: verbal learning. In line with this view, the most robust relationships with reading ability have been consistently observed in PAL tasks with nonword stimuli (Elbro \& Jensen, 2005; Hulme et al., 2007; Mayringer \& Wimmer, 2000; Vellutino et al., 1975). As outlined in the introduction, the role of verbal learning in reading development is supported by emerging evidence that novel phonological representations (i.e., spelling pronunciations) are generated through phonological recoding and subsequently utilized in word recognition (Elbro, et al., 2012; Ranbom \& Connine, 2011; Tunmer \& Chapman, 2011). According to this framework, the relationship between verbaloutput PAL and reading ability may be explained by the common demand on verbal learning.

In support of this view, the pattern of relationships between verbal-output PAL and reading accuracy and fluency parallels the findings of Elbro et al. (2012), in which word recognition from spelling pronunciations better accounted for individual differences in reading accuracy than fluency. If spelling pronunciations aid in the arrival at the correct spoken representation in the lexicon, it follows that it is the ability to learn these pronunciations and utilize them in word recognition that determines success. In other words, so long as the spelling pronunciation cues the selection of the correct word, the efficiency with which one arrives at the word is irrelevant. Thus, we might expect a stronger relationship with reading accuracy than 
fluency, because fluency measures are compounded by both accuracy and speed (the latter being unnecessary for verbal learning).

However we caution against the over-interpretation of our results regarding accuracy and fluency. Verbal-output PAL only reached significance as a predictor of accuracy, but the difference in the variance explained by verbal-output PAL in reading fluency vs. accuracy was not statistically significant. Further investigation of the relationship between verbal-output PAL and measures of fluency and accuracy is therefore warranted.

Finally, it is important to consider whether verbal learning might be more distally related to reading ability, rather than playing a more integral or direct role. For example, it may be that performance on verbal-output PAL is a reflection of the level of support available from existing orthographic knowledge to support verbal learning. On this view, more skilled readers might be better able to learn new phonological forms in a verbal-output PAL task due to orthographic information being activated automatically, even though the tasks do not involve orthographic knowledge directly (e.g., Metsala, 1997; Nation \& Hulme, 2011; Ricketts, Bishop \& Nation, 2009). In line with this, Lervåg et al. (2009) found that visual-verbal PAL did not emerge as a longitudinal predictor of reading skill when measured in pre-readers. Additionally, children with more precise and segmented phonological representations may be better able to form novel phonological representations and can thus more easily attach these forms to orthographic information (de Jong, Seveke, \& van Veen, 2000; Messbauer \& de Jong, 2003. Taken together, these findings suggest that verbal learning ability (as indexed by PAL) may be a consequence, rather than a cause, of reading development. However, the current data do not allow us to speak to this issue; 
additional longitudinal and cross-sectional studies are needed to disentangle the causal and reciprocal relationships between verbal-output PAL and reading development.

In summary, the results of the present study add to a growing body of research indicating that the PAL-reading relationship is both robust, and independent from phoneme deletion and RAN. Additionally, our results shed new light on the locus of the PAL-reading relationship by disentangling the role of crossmodal and verbal demands. Contrary to the predictions of the crossmodal hypothesis, we found that the PAL-reading relationship is not specific to visual-verbal PAL, but instead, emerges for PAL tasks with a verbal output component. 


\section{References}

Byrne, B. J. (1998). The Foundation Of Literacy: The Child's Acquisition Of The Alphabetic Principle. Psychology Press.

Davis M. H., Di Betta A. M., Macdonald M. J. E., \& Gaskell M. G. (2008). Learning and consolidation of novel spoken words. Journal of Cognitive Neuroscience , 21, 803820.

de Jong, P.F., \& van der Leij, A. (1999). Specific contributions of phonological abilities to early reading acquisition: Results from a Dutch latent variable longitudinal study. Journal of Educational Psychology, 91(3), 450-476.

de Jong, P. F., Seveke, M.-J., \& van Veen, M. (2000). Phonological Sensitivity and the Acquisition of New Words in Children. Journal of Experimental Child Psychology, 76(4), 275-301.

Dumay, N., \& Gaskell, M.G. (2007) Sleep-associated changes in the mental representation of spoken words. Psychological Science, 18(1), 35-39.

Elbro, C. (1998). When reading is "readn" or somthn. Distinctness of phonological representations of lexical items in normal and disabled readers. Scandinavian Journal of Psychology, 39(3), 149-153.

Elbro, C., de Jong, P. F., Houter, D., \& Nielsen, A. M. (2012): From spelling pronunciation to lexical access: A second step in word decoding? Scientific Studies of Reading, 16(4),341-349.

Elbro, C., \& Jensen, M. N. (2005). Quality of phonological representations, word learning, and phoneme awareness in dyslexic and normal readers. Scandinavian Journal of Psychology, 46, 375-384. 
Foster, H. (2007) Single Word Reading Test 6-16, GL Assessment Limited.

Harm, M. W., \& Seidenberg, M. S. (1999). Phonology, reading acquisition, and dyslexia: Insights from connectionist models. Psychological Review, 106(3), 491-528.

Hulme, C., Goetz, K., Gooch, D., Adams, J., \& Snowling, M. J. (2007). Paired-associate learning, phoneme awareness, and learning to read. Journal of Experimental Child Psychology, 96(2), 150-166.

Lervåg, A., Bråten, I., \& Hulme, C. (2009). The cognitive and linguistic foundations of early reading development: A Norwegian latent variable longitudinal study. Developmental Psychology, 45(3), 764-781.

Mayringer, H. \& Wimmer, H. (2000). Pseudoname learning by German-speaking children with dyslexia: Evidence for a phonological learning deficit. Journal of Experimental Child Psychology, 75, 116-133.

Melby-Lervåg, M., Lyster, S.-A. H., \& Hulme, C. (2012). Phonological skills and their role in learning to read: A meta-analytic review. Psychological Bulletin, 138(2), 322-352.

Messbauer, V. C. ., \& de Jong, P. F. (2003). Word, nonword, and visual paired associate learning in Dutch dyslexic children. Journal of Experimental Child Psychology, 84(2), 77-96.

Metsala, J. L. (1997). Spoken word recognition in reading disabled children. Journal of Educational Psychology, 89(1), 159-169.

Muter, V., Hulme, C., Snowling, M. J., \& Stevenson, J. (2004). Phonemes, Rimes, Vocabulary, and Grammatical Skills as Foundations of Early Reading Development: Evidence From a Longitudinal Study. Developmental Psychology, 40(5), 665-681. 
Muthén, L. K., \& Muthén, B. O. (2009). Mplus (Version 5.21). Los Angeles: Muthén \& Muthén

Nation, K., Hulme, C. (2011). Learning to read changes children's phonological skills: Evidence from a latent variable longitudinal study of reading and nonword repetition. Developmental Science. 14, 649-659

Norton, E. S., \& Wolf, M. (2012). Rapid Automatized Naming (RAN) and Reading Fluency: Implications for Understanding and Treatment of Reading Disabilities. Annual Review of Psychology, 63(1), 427-452.

Ramus, F., \& Szenkovits, G. (2008). What phonological deficit? The Quarterly Journal of Experimental Psychology, 61(1), 129-141.

Rastle, K., Harrington, J., \& Coltheart, M. (2002). 358,534 nonwords: The ARC Nonword Database. The Quarterly Journal of Experimental Psychology Section A, 55(4), 13391362.

Ricketts, J., Bishop, D. V. M., \& Nation, K. (2009). Orthographic facilitation in oral vocabulary acquisition. The Quarterly Journal of Experimental Psychology, 62(10), 1948-1966.

Schatschneider, C., Fletcher, J. M., Francis, D. J., Carlson, C. D., \& Foorman, B. R. (2004). Kindergarten Prediction of Reading Skills: A Longitudinal Comparative Analysis. Journal of Educational Psychology, 96(2), 265-282.

Schneider, W., Eschman, A, \& Zuccolotto, A. (2007). E-Prime: User's Guide, Version 2.0. Psychology Software Tools. 
Seidenberg, M. S. McClelland, J. L. (1989). A distributed, developmental model of word recognition and naming. Psychological Review, 96, 523-568.

Snowling, M., Hulme, C. (1994). The development of phonological skills in children. Philosophical Transactions of the Royal Society of London B346, 21-27

Stevens, J. Applied multivariate statistics for the social sciences. 5th ed. London: Routledge; 2009

Swan, D., \& Goswami, U. (1997). Phonological Awareness Deficits in Developmental Dyslexia and the Phonological Representations Hypothesis. Journal of Experimental Child Psychology, 66(1), 18-41.

Torgesen, J.K., Wagner, R. K., \& Rashotte, C.A. (1999). Test of Word Reading Efficiency. Austin, TX: PRO-ED Publishing, Inc.

Truman, A., \& Hennessey, N. W. (2006). The locus of naming difficulties in children with dyslexia: Evidence of inefficient phonological encoding. Language and Cognitive Processes, 21(4), 361-393.

Vellutino, F. R., Fletcher, J. M., Snowling, M. J., \& Scanlon, D. M. (2004). Specific reading disability (dyslexia): what have we learned in the past four decades? Journal of Child Psychology and Psychiatry, 45(1), 2-40.

Vellutino, F. R., Scanlon, D. M., \& Spearing, D. (1995). Semantic and Phonological Coding in Poor and Normal Readers. Journal of Experimental Child Psychology, 59(1), 76-123. 
Vellutino, F. R., Steger, J. A., Harding, C. J., \& Phillips, F. (1975). Verbal vs non-verbal paired-associates learning in poor and normal readers. Neuropsychologia, 13(1), 7582.

Wagner, R. K., Torgesen, J. K., and Rashotte, C. A. (1999). The Comprehensive Test of Phonological Processing (CTOPP). Pro-Ed Inc.

Warmington, M., \& Hulme, C. (2011). Phoneme Awareness, Visual-Verbal PairedAssociate Learning, and Rapid Automatized Naming as Predictors of Individual Differences in Reading Ability. Scientific Studies of Reading, 16(1), 45-62.

Wechsler, D. ( 1999 ). Wechsler Abbreviated Scale of Intelligence . San Antonio, TX : The Psychological Corporation

Wimmer, H., Mayringer, H., \& Landerl, K. (1998). Poor Reading: A Deficit in SkillAutomatization or a Phonological Deficit? Scientific Studies of Reading, 2(4), 321340.

Windfuhr, K. L., \& Snowling, M. J. (2001). The Relationship between Paired Associate Learning and Phonological Skills in Normally Developing Readers. Journal of Experimental Child Psychology, 80(2), 160-173. 
Table 1

Means (and standard deviations) for measures of PAL, phoneme awareness, $R A N$, and reading

\begin{tabular}{|c|c|c|c|}
\hline & Mean & $S D$ & Range \\
\hline Phoneme Deletion $^{\mathrm{a}}$ & 9.78 & 2.81 & $5-15$ \\
\hline RAN Digits $^{a}$ & 9.95 & 2.51 & $6-15$ \\
\hline $\begin{array}{l}\text { TOWRE Sight Word } \\
\text { Efficiency }^{\mathrm{b}}\end{array}$ & 104.67 & 11.59 & $76-133$ \\
\hline TOWRE Nonword Efficiency ${ }^{b}$ & 104.33 & 14.01 & 74-139 \\
\hline Single Word Reading Test ${ }^{\mathrm{b}}$ & 96.84 & 13.59 & $69-129$ \\
\hline Visual-verbal PAL $^{\mathrm{c}}$ & 5.84 & 5.22 & $0-27$ \\
\hline Verbal-verbal PAL ${ }^{\mathrm{c}}$ & 4.86 & 4.62 & $0-25$ \\
\hline Visual-visual $\mathrm{PAL}^{\mathrm{c}}$ & 9.98 & 4.72 & $1-20$ \\
\hline Verbal-visual PAL ${ }^{\mathrm{c}}$ & 11.61 & 5.40 & $3-25$ \\
\hline
\end{tabular}

$15 ;{ }^{\mathrm{c}}$ Raw score, $\max =30$ 
Table 2

Correlations among PAL, RAN, and reading measures (simple correlations above the diagonal, partial correlations controlling for age below the diagonal)

\begin{tabular}{|c|c|c|c|c|c|c|c|c|c|}
\hline & 1 & 2 & 3 & 4 & 5 & 6 & 7 & 8 & 9 \\
\hline 1. Phoneme Deletion & & $-.29^{*}$ & $.45^{* *}$ & $.52 * *$ & $.62^{*}$ & $.31 *$ & $.31 *$ & .14 & $.25 *$ \\
\hline 2. RAN & -.18 & & $-.70 * *$ & $-.67 * *$ & $-.56 * *$ & -.14 & -.26 & -.16 & -.20 \\
\hline 3. Sight Word Fluency & $.36^{* *}$ & $-.61 * *$ & & $.79^{* *}$ & $.74 * *$ & $.33^{* *}$ & $.30^{*}$ & .10 & .21 \\
\hline 4. Nonword Fluency & $.47 * *$ & $-.63 * *$ & $.77 * *$ & & $.71 * *$ & $.32 *$ & $.30 *$ & .13 & .15 \\
\hline 5. Word Reading Accuracy & $.58 * *$ & $-.45 * *$ & $.67 * *$ & $.68 * *$ & & $.39 * *$ & $.42 * *$ & .13 & .17 \\
\hline 6. Visual-Verbal PAL & $.28 *$ & -.05 & $.27 *$ & $.27 *$ & $.34 * *$ & & $.45^{* *}$ & $.31 *$ & $.33^{* *}$ \\
\hline 7. Verbal-Verbal PAL & $.26^{*}$ & -.20 & .23 & $.25^{*}$ & $.37 * *$ & $.42 * *$ & & $.41^{* *}$ & $.34 * *$ \\
\hline 8. Visual-Visual PAL & .12 & -.13 & .07 & .10 & .10 & $.29 *$ & $.40 * *$ & & .31 \\
\hline 9. Verbal-Visual PAL & .19 & -.10 & .10 & .08 & .06 & $.29 *$ & $.30 *$ & $.30 *$ & \\
\hline
\end{tabular}

$* p<.05 . * p<.01$. 
Figure Captions

Figure 1. Path analysis models predicting word reading fluency, nonword reading fluency, and word reading accuracy from phoneme deletion, $R A N$, and $(A)$ visual-verbal $P A L,(B)$ verbal-verbal $P A L,(C)$ verbal-visual PAL, or (D) visual-visual PAL. Solid arrows represent statistically significant predictive relationships. Dashed arrows represent non-significant predictive relationship: $(a) p=.059,(b) p=.086$, (c) $p=.498,(d) p=.459,(e) p=.981,(f) p=.595,(g) p=.466,(h) p=.665,(i) p=.872,(j) p=.907$. Double-headed arrows represent correlations between predictors. The arrows and values above the dependent variables represent the proportion of variance not explained by the predictors in the model, or the residual variance. All values presented in the model are standardized values.

Figure 2. Structural equation model of the relationship between the latent cognitive constructs and reading ability. Circles represent latent variables. Rectangles to the left of the diagram represent observed indicators for the latent variables, and rectangles to the right of the diagram represent observed dependent variables. Solid arrows represent statistically significant predictive relationships. Dashed arrows represent non-significant predictive relationships. Parallel lines indicate that the paths were constrained to be equal. The arrows and values above the dependent variables represent the proportion of variance not explained by the predictors in the model, or the residual variance. All values presented in the model are standardized values. 
A

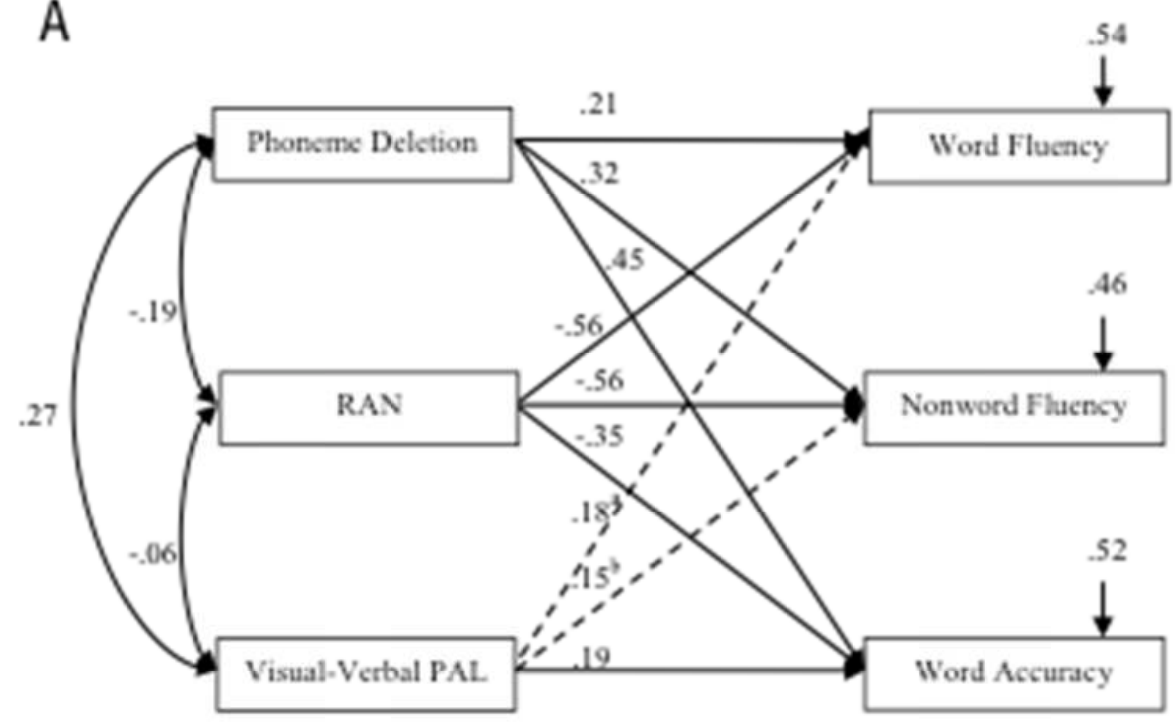

C

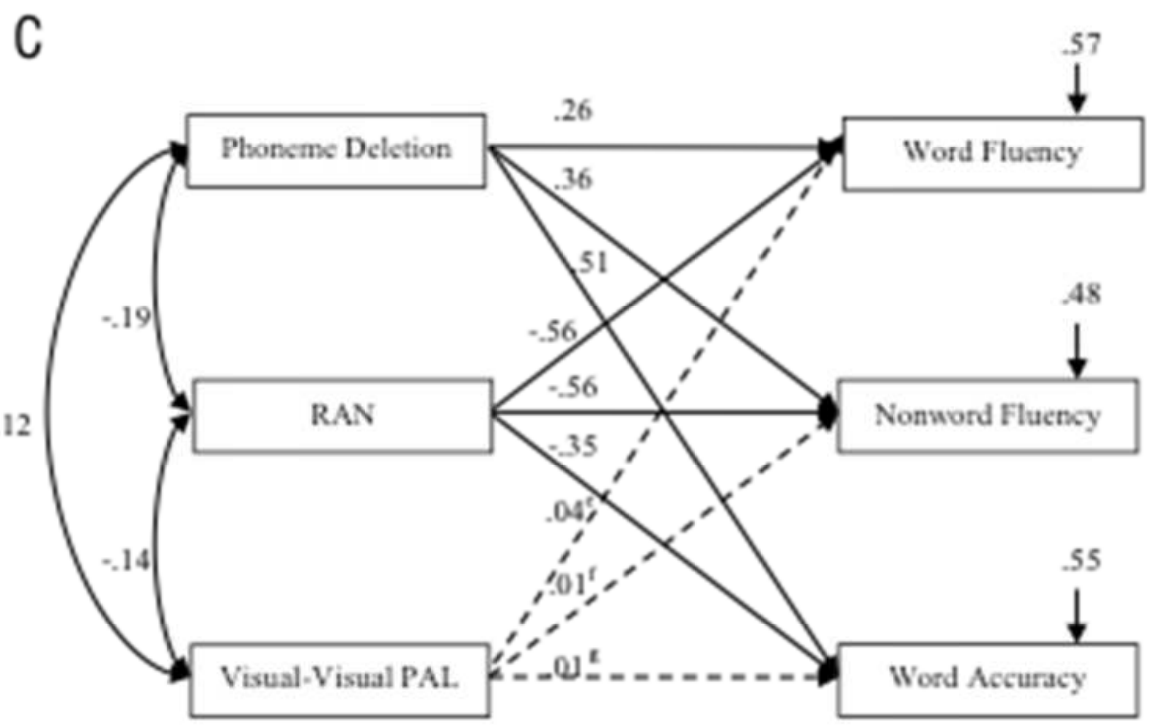

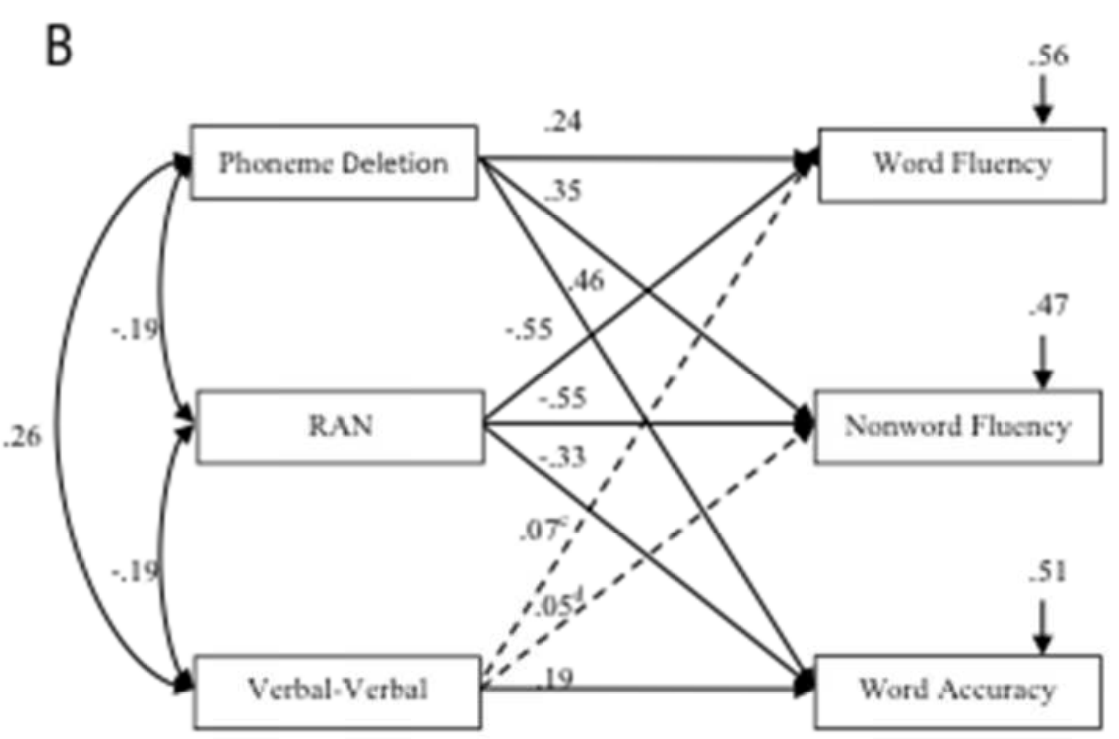

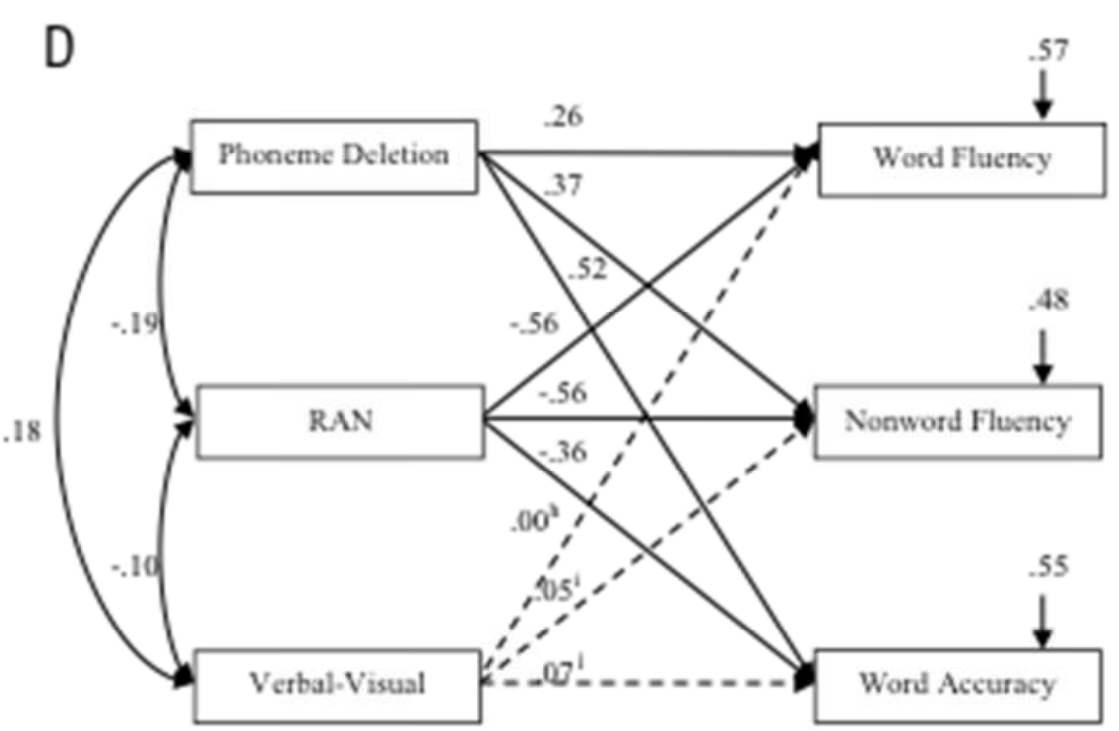




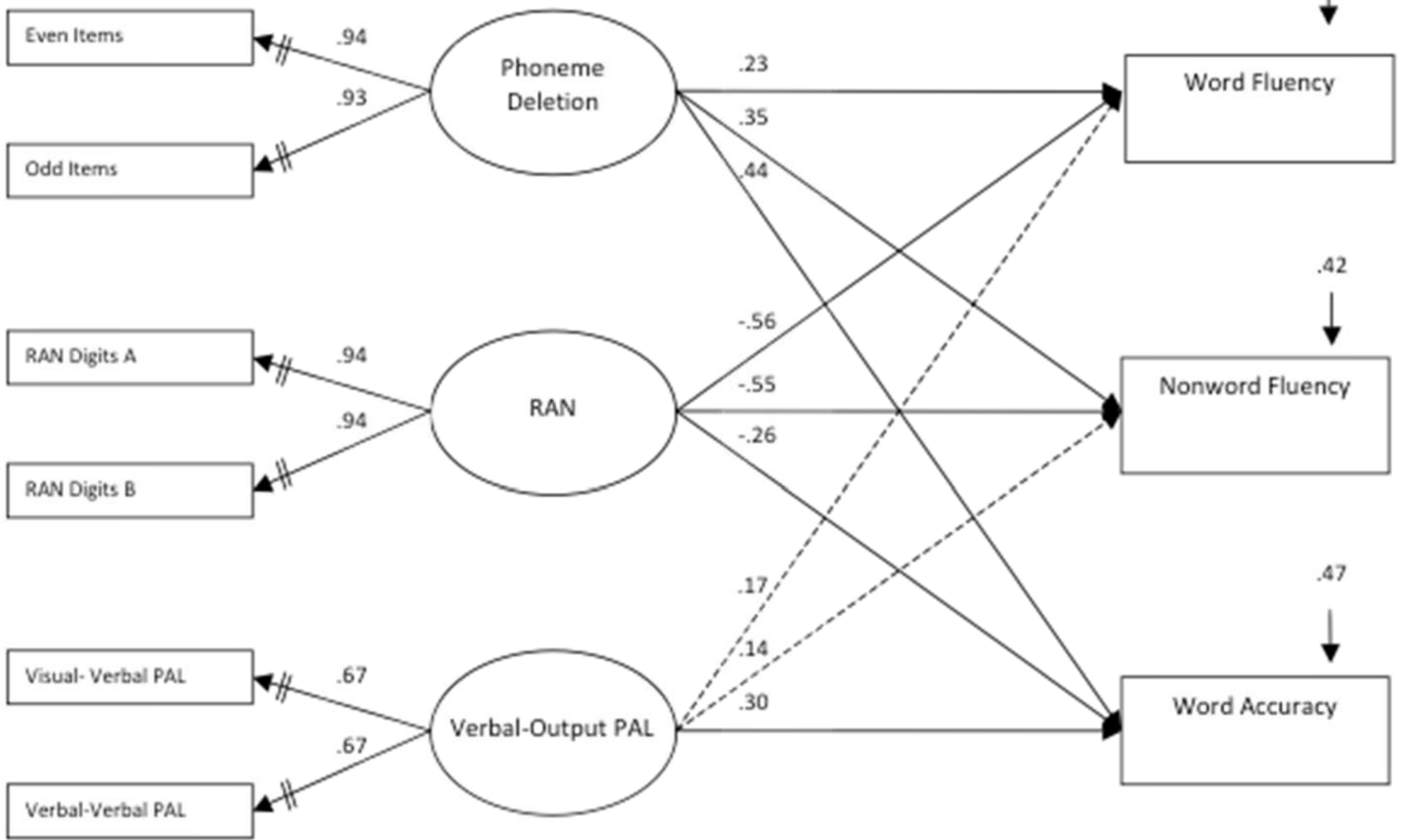


Cognitive Demands in Paired Associate Learning /35 